\title{
Avaliação da exposição ao Pb e Cd em crianças de 0 a 17 anos por consumo de alimentos vegetais cultivados em solos contaminados no município de Santo Amaro (BA)
}

\author{
Evaluation of $\mathrm{Pb}$ and $\mathrm{Cd}$ exposure in children $\mathrm{O}$ to 17 years \\ of age by consumption of vegetable food cultivated \\ in soils contaminated in Santo Amaro (BA)
}

\section{Gustavo Alonso Muñoz Magna', Sandro Lemos Machado², Roberto Bagattini Portella ${ }^{3}$, Miriam de Fátima Carvalho ${ }^{4}$}

\section{RESUMO}

O objetivo do presente trabalho foi estimar as doses de exposição aos contaminantes chumbo (Pb) e cádmio (Cd)em crianças de Oa17anos de idade via consumo de alimentos vegetais cultivados em quintais contaminados, localizados no entorno de uma metalúrgica desativada, no município de Santo Amaro, Estado da Bahia. Para a estimativa das doses foi utilizada a metodologia de avaliação de riscos sobre a saúde humana, especificamente a etapa de análise da exposição, determinando as concentrações dos metais e variáveis sociodemográficas das crianças. As doses de exposição estimadas para ambos os contaminantes superaram os valores recomendados pela Organização Mundial da Saúde (OMS),

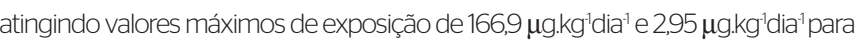
o Pb e o Cd, respectivamente, por consumo de frutas. Os resultados comprovam que o consumo de alimentos vegetais cultivados em quintais pela população residente próxima à antiga fábrica é uma importante rota de exposição atual aos metais, representando um risco para a saúde da população.

Palavras-chave: exposição; avaliação de riscos; metais; contaminação do solo; vegetais; passivo ambiental.

\section{ABSTRACT}

This paper aims to estimate the exposure of children between $\mathrm{O}$ to 17 years old to lead $(\mathrm{Pb})$ and cadmium ( $\mathrm{Cd}$ ) via ingestion of vegetables cultivated in contaminated backyards located in the surroundings of a deactivated lead metallurgy in Santo Amaro, Bahia, Brazil. For the estimation of the exposure doses, the risk assessment on human health methodology, exposure analysis, was used, determining the concentrations of metals and socio-demographic variables of children The estimated doses for both contaminants exceeded the values recommended by the World Health Organization (WHO). Maximum obtained values were $166.9 \mu \mathrm{g} \mathrm{kg}^{-1} \mathrm{day}^{-1}$ and $2.95 \mu \mathrm{g} . \mathrm{kg}^{-1} \mathrm{day}{ }^{-1}$ for $\mathrm{Cd}$ and $\mathrm{Pb}$, respectively, due to fruits consumption. The results show that the consumption of vegetables cultivated in the backyards by the population living near the deactivated metallurgical plant plays an important role in the exposition of the population to metals.

Keywords: exposure; risk assessment; metals; soil contamination; vegetables; environmental liabilities.

'Engenheiro Ambiental, Doutorando em Energia e Ambiente, Departamento de Ciência e Tecnologia dos Materiais da Escola Politécnica da Universidade Federal da Bahia (UFBA) Salvador (BA), Brasil.

2Engenheiro Civil, Doutor em Geotecnia, Professor da Escola Politécnica da UFBA - Departamento de Ciência e Tecnologia dos Materiais da Escola Politécnica da Universidade Federal da Bahia (UFBA) - Salvador (BA), Brasil.

3Engenheiro Civil, Doutor em Engenharia Ambiental, Professor no Centro das Ciências Exatas e das Tecnologias da Universidade Federal do Oeste da Bahia (UFOB) Barreiras (BA), Brasil.

«Engenheira Civil, Doutora em Geotecnia, Professora da Universidade Católica do Salvador (UCSAL) - Escola de Engenharia da Universidade Católica do Salvador (UCSAL) - Salvador (BA), Brasil.

Endereço para correspondência: Gustavo Alonso Muñoz Magna - Rua Aristides Novis, 2, Federação - 04021-630 - Salvador (BA), Brasil - E-mail: ingmag@gmail.com

Fonte de financiamento: Coordenação de Aperfeiçoamento de Pessoal de Nível Superior (CAPES), Fundação Escola Politécnica e Secretaria de Ciência, Tecnologia e Inovação do Estado da Bahia (SECTI-BA).

Recebido: 19/06/12 - Aceito: 16/01/14 - Reg. ABES: 430 


\section{INTRODUÇÃO}

Historicamente, uma das ideias que mais contribuiu para a formação de enormes passivos ambientais é a de que os compostos e elementos químicos produzidos de forma antrópica e posteriormente disponibilizados para o meio ambiente poderiam ser assimilados pela natureza, transformando-os em substâncias inócuas, ou poderiam ser diluídos de forma a não representar ameaça para o homem e os ecossistemas (PORTELLA, 2007). Esse pressuposto foi assumido em um passado não muito distante e com maior força e frequência no solo, compartimento ambiental considerado à época como um meio com capacidade praticamente ilimitada para acumular contaminantes e armazenar resíduos sem gerar efeitos deletérios imediatos (TOBAR, 2006).

Alguns fatos com consequências trágicas para a saúde da população, como o caso do Love Canal no estado de Nova York nos EUA em 1978 e também no Brasil, tais como os acontecidos no Vale do Ribeira, entre os Estados de São Paulo e Paraná (PAOLIELLO et al., 2002), Cubatão (CUNHA et al., 2005) e Bauru (FREITAS et al., 2007), no Estado de São Paulo, dentre outros, colocaram em evidência o equívoco desta suposição e a relativa facilidade com que a carga contaminante do solo pode ser transferida a outros meios mais sensíveis, com um elevado potencial de efeitos negativos sobre a saúde humana.

Durante o período de 1956 a 1993, o município de Santo Amaro, Bahia, foi sede de atividades metalúrgicas para o beneficiamento do chumbo, que resultaram na produção de escórias e outros resíduos com cerca de $4 \%$ em peso de óxido de chumbo $(\mathrm{PbO})$ e traços de cádmio $(\mathrm{Cd})$, antimônio (Sb) e arsênio (As), entre outros metais (RIBEIRO et al., 2003).

Devido à falta de conhecimento, ao gerenciamento inadequado dos resíduos por parte da Companhia Brasileira de Chumbo (COBRAC) e à regulamentação ineficaz durante o período de funcionamento da fábrica, os resíduos do processo metalúrgico foram utilizados de diversas formas pelo próprio poder público e pela população, como por exemplo, na pavimentação das ruas da cidade e como aterro em quintais de casas. Os volumes de escória dispostos sob a pavimentação das ruas e quintais além da área entorno da fábrica foram estimados em aproximadamente $55.000 \mathrm{~m}^{3}$ e $180.000 \mathrm{~m}^{3}$, respectivamente (MACHADO et al., 2004). Ainda, resultados obtidos mediante testes de lixiviação demonstraram que a escória produzida pela ex-fábrica corresponde a um resíduo classe I (resíduo perigoso), conforme norma brasileira 10.004/1987 (ANJOS, 2003; MACHADO et al., 2004).

Além da inadequada disposição e utilização da escória, a área circunvizinha em um raio de aproximadamente $4 \mathrm{~km}$ foi atingida com intensidade por emissões atmosféricas produto da operação por 37 anos da fábrica. Estas emissões foram acumulativas e emitiram principalmente material particulado composto por metais, tais como $\mathrm{Pb}, \mathrm{Cd}, \mathrm{As}$ e Zn. No ano de 2003 a situação da contaminação na área urbana foi reportada pela Fundação Nacional de Saúde, que identificou o município de Santo Amaro como uma das áreas prioritárias para a vigilância ambiental relacionada aos solos contaminados no Brasil (FUNASA, 2003). Esses fatos e suas posteriores consequências conformaram um quadro de contaminação importante no cenário brasileiro.

Transcorridos 20 anos do encerramento das operações da metalúrgica, antecedentes referidos ao solo confirmaram a persistência da contaminação por metais em solos de quintais do município e nos arredores da antiga fábrica. Das análises realizadas em 223 amostras de solo superficial da Rua Rui Barbosa (principal local atingido pelo uso indiscriminado de escória), aproximadamente 80\% apresentaram valores acima do limite de investigação para o chumbo para áreas residenciais (300 mg.kg ${ }^{-1}$ ) e 50\% apresentaram concentrações acima do limite de investigação para áreas industriais (900 mg.kg-1), ambos estabelecidos pela resolução CONAMA nº420/2009 (RABELO, 2010; MACHADO et al., 2013; BRASIL, 2009).

Solos com altos níveis de contaminação por metais podem implicar em riscos à saúde da população devido à sua absorção pelas plantas e sua potencial transferência através da cadeia alimentar. Desta forma, os contaminantes presentes no solo podem seguir diferentes rotas até alcançar os seres humanos, como a ingestão de alimentos contaminados, uma das principais vias de exposição ao $\mathrm{Pb}$ e $\mathrm{Cd}$ (AGENCY FOR TOXIC SUBSTANCES AND DISEASE REGISTRY, 2007; KABATA-PENDIAS \& MUKHERJEE, 2007). Neste cenário, a contaminação de solos por metais na cidade de Santo Amaro, Bahia, é um aspecto de grande preocupação quanto aos riscos sobre a saúde da população devido principalmente a três fatores. O primeiro corresponde à persistência deste tipo de contaminação no solo; o segundo, à toxicidade destes elementos; e o terceiro, à presença de população potencialmente atingida, especificamente crianças, que são a população mais susceptível aos efeitos dos metais. Este problema se reveste de uma maior relevância porque os vegetais constituem uma parte importante da dieta humana devido ao seu conteúdo de carboidratos, proteínas, vitaminas, minerais e elementos traços ou essencialmente biológicos e torna-se ainda mais complexo devido ao fato de que metais tais como o $\mathrm{Pb}$ e o $\mathrm{Cd}$ não têm uma função biológica conhecida, sendo, portanto, bioacumuláveis nos seres humanos (CHARY et al., 2008; KABATA-PENDIAS \& PENDIAS, 2001).

O objetivo do presente trabalho foi estimar as doses de exposição aos metais $\mathrm{Pb}$ e $\mathrm{Cd}$ em crianças de 0 a 17 anos de idade pelo consumo de alimentos vegetais cultivados em quintais com altos níveis de contaminação localizados próximos a uma metalúrgica desativada, no município de Santo Amaro, Bahia.

\section{Efeitos sobre a saúde humana e valores de referência para ingestão dos contaminantes $\mathrm{Pb}$ e $\mathrm{Cd}$ mediante alimentos vegetais}

$\mathrm{O} \mathrm{Pb}$ e o $\mathrm{Cd}$ são considerados elementos tóxicos não essenciais que se acumulam no organismo humano. São amplamente conhecidos os impactos 
à saúde que podem ser desenvolvidos como resultado da excessiva acumulação destes metais no corpo humano. Em relação ao $\mathrm{Pb}$, a toxicidade desse contaminante gera desde efeitos claros, ou clínicos, até efeitos sutis, ou bioquímicos (MOREIRA \& MOREIRA, 2004). Os efeitos tóxicos do $\mathrm{Pb}$ são conhecidos há séculos, porém, somente nas últimas décadas tem se demonstrado que níveis de exposição considerados comumente como baixos podem estar associados a efeitos adversos nos seres humanos, atingindo principalmente o sistema nervoso em desenvolvimento, o sistema hematológico e o cardiovascular, além do rim, quando a exposição é excessiva e prolongada. Associado a isso, a International Agency for Research on Cancer (IARC), classificou o Pb inorgânico e os compostos de $\mathrm{Pb}$ como possíveis cancerígenos para humanos (Grupo 2B) e a Agency for Toxic Substances and Disease Registry (ATSDR), na sua lista prioritária de substâncias perigosas, classificou o $\mathrm{Pb}$ no segundo lugar em função da sua farta ocorrência em sítios contaminados e sua toxicidade. Complementando, devido aos múltiplos modos de ação do $\mathrm{Pb}$ nos sistemas biológicos, esse elemento poderia afetar potencialmente qualquer sistema ou órgão no corpo humano (AGENCY FOR TOXIC SUBSTANCES AND DISEASE REGISTRY, 2007).

Tanto as crianças quanto os adultos são suscetíveis aos efeitos na saúde por exposição ao metal, entretanto, as vias de exposição e os efeitos podem ser bastante diferentes e se faz importante distinguir entre adultos e crianças devido à diferença de suscetibilidade entre esses dois grupos (MOREIRA \& MOREIRA, 2004). Crianças estão mais expostas em regiões apresentando contaminação ambiental devido ao seu comportamento e fisiologia (CUNHA, 2003). A sensibilidade das crianças aos efeitos da exposição se dá principalmente devido aos seguintes aspectos: (a) o consumo por quilo de massa é maior do que nos adultos; (b) crianças colocam na boca objetos que estão no chão com maior frequência; (c) a absorção do $\mathrm{Pb}$ pelo organismo da criança é maior quando comparado com adulto e; (d) crianças pequenas estão em desenvolvimento rápido e constante, tornando-se mais vulneráveis aos efeitos do metal. É importante destacar que possivelmente não exista um valor limite para as concentrações de chumbo no organismo humano onde, a partir deste, se comece a observar os efeitos adversos na saúde das crianças (AGENCY FOR TOXIC SUBSTANCES AND DISEASE REGISTRY, 2007).

Os principais efeitos tóxicos devido ao excesso de Cd nos seres humanos se relacionam com danos nos rins, hipertensão, enfisema, malformações e diminuição da função reprodutora (KABATA-PENDIAS \& MUKHERJEE, 2007). Vale destacar que o órgão alvo no qual o cádmio exerce sua toxicidade depende do tipo de exposição. Porém, ao falar de exposição crônica ao Cd, o principal órgão atingido é o rim. O Cd pode se acumular nos rins se ingerido em níveis baixos e durante longos períodos de tempo. Se essa acumulação nos rins chega a um nível suficientemente alto, pode haver danos renais e deterioramento ósseo. Comumente os efeitos observados em adultos expostos ao Cd são também observados nas crianças, e exposições ainda que em baixas quantidades podem ter consequências a longo prazo. Isto se deve principalmente à característica acumulativa do Cd e sua longa vida média no organismo (AGENCY FOR TOXIC SUBSTANCES AND DISEASE REGISTRY, 2012). O Cd e seus compostos são considerados tóxicos e desde 1993 são classificados como pertencentes ao Grupo 1 pela IARC, sendo o Cd definido como possuidor de evidências suficientes de carcinogenecidade em humanos. Complementarmente, a ATSDR na sua lista prioritária de substâncias perigosas classificou o Cd no sétimo lugar em função da sua ocorrência em sítios contaminados e sua toxicidade (AGENCY FOR TOXIC SUBSTANCES AND DISEASE REGISTRY, 2012).

Em relação aos valores de referência, a Organização Mundial de Saúde e a Organização das Nações Unidas para a Agricultura e Alimentação (OMS/FAO), através da Comissão Codex Alimentar (OMS, 2009), definiu valores de Ingestão Semanal Tolerável Provisória (ISTP) ou Ingestão Diária Admissível (IDA) como doses permissíveis e de segurança, em cenários provisórios, para contaminantes metálicos presentes em alimentos. $\mathrm{O}$ valor do nível toxicológico de segurança definido pela OMS/FAO para o $\mathrm{Pb}$ foi estabelecido como ISTP $=25 \mu \mathrm{g} \cdot \mathrm{kg}^{-1}$. semana $^{-1}$ ou IDA $=3,5 \mu \mathrm{g} \cdot \mathrm{kg}^{-1} \cdot \mathrm{dia}^{-1}$. No caso do Cd, os valores atuais são de ISTP $=7,0 \mu \mathrm{g} \cdot \mathrm{kg}^{-1}$. semana ${ }^{-1}$ ou IDA $=1,0 \mu \mathrm{g} \cdot \mathrm{kg}^{-1} \cdot \mathrm{dia}^{-1}$. Os valores estabelecidos pela OMS/FAO consideram a ingestão dos contaminantes no total da dieta alimentar e estão referidos a adultos. Outras instituições internacionais tais como a ATSDR, através dos Minimal Risk Levels (MRLs), e a United States Environmental Protection Agency (USEPA), mediante a Reference dose (RfD), têm definido doses de referência para contaminantes metálicos. Contudo, optou-se pela utilização dos valores estabelecidos pela OMS/FAO por apresentar valores de segurança para ambos os contaminantes de interesse e por estarem referidos a alimentos. Para fins de comparação dos resultados deste estudo, também são apresentados valores de doses de exposição obtidos por diferentes autores e locais através do consumo de alimentos vegetais.

\section{METODOLOGIA}

\section{Área de estudo e campanhas de investigação em campo}

A área de estudo compreendeu principalmente os quintais com presença de hortas e plantas com frutas comestíveis da Rua Rui Barbosa, localizados entre as coordenadas UTM (8613602; 529908 e 8613012; 531037), local identificado no projeto PURIFICA no ano 2003 como uma das áreas prioritárias de investigação para mitigação do problema de contaminação do solo em Santo Amaro (MACHADO, 2003). Na Figura 1 se apresenta a área de estudo.

Ainda, a escolha definitiva desta rua levou em consideração a sua localização (proximidade à antiga fábrica), o histórico de utilização da escória como aterro em quintais e como base da pavimentação. 


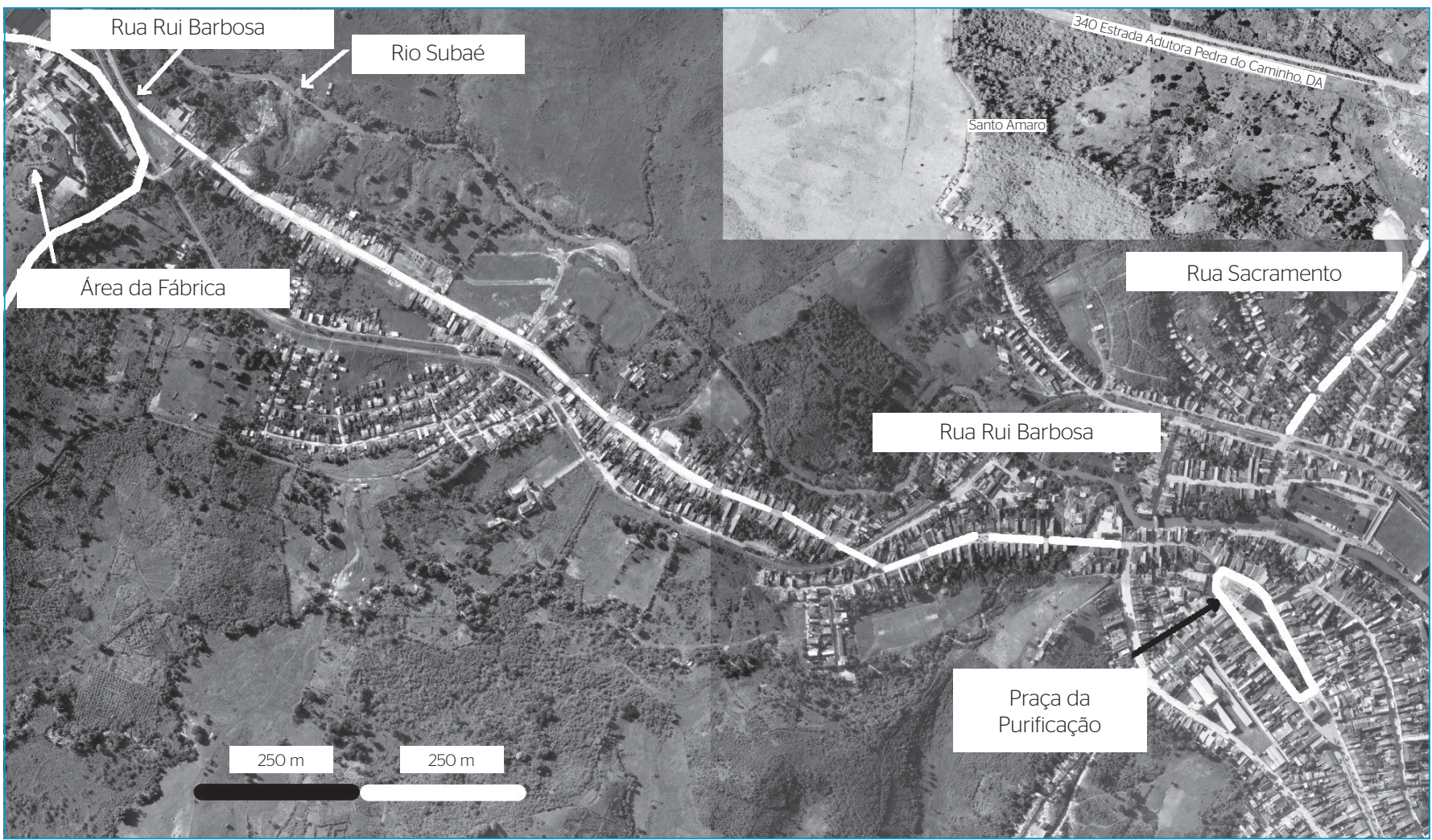

Figura 1 - Área de estudo.

As atividades de campo constaram, preliminarmente, da identificação e reconhecimento da área, além do levantamento de dados. Nesta etapa, foram visitadas as instalações da antiga fábrica e suas proximidades. Na área urbana se interagiu com os moradores da Rua Rui Barbosa, primeiramente informando sobre o intuito da pesquisa, além de solicitar a sua participação na mesma. Logo da aceitação da participação, o responsável pela residência e as crianças assinaram um termo de consentimento livre esclarecido. Após a formalização da participação foram aplicados questionários padronizados específicos ao objeto de estudo e cuja finalidade preliminar era a identificação das espécies comestíveis presentes nos quintais, sua abundância e a obtenção da frequência de consumo das mesmas pela população (vezes na semana).

Após obtidos os resultados dos questionários e especificamente o inquérito sobre presença de alimentos vegetais em quintais, os mesmos foram quantificados determinando-se as frequências de ocorrência de cada vegetal encontrado no total dos quintais estudados. Da mesma forma, foi obtido o valor de frequência de consumo dos vegetais pela população utilizando como parâmetro estatístico a moda. Os alimentos vegetais foram selecionados conforme a sua ocorrência e abundância nos quintais, além da sua frequência de consumo e corresponderam a 13 espécies de frutas e ervas, tais como acerola (Malpichia glabral), aroeira (Schinus molle), alumã (Veronia bahiensis tol), banana (Musa paradisica), entre outros.

\section{Amostragem e determinação dos contaminantes nos alimentos vegetais de estudo}

A coleta de amostras vegetais foi realizada de forma manual nos quintais selecionados sendo colhido um mínimo de três frutos para cada espécie vegetal segundo a disponibilidade no local. As amostras de vegetais coletadas foram acondicionadas em sacos plásticos fechados e devidamente etiquetados para seu posterior envio para o laboratório. Já no laboratório, as amostras foram congeladas até o momento da realização das análises. Para mensurar os conteúdos dos contaminantes nas espécies vegetais foi necessário dividir o processo em três etapas: (1) acondicionamento e preparo de amostras; (2) abertura das amostras (digestão ácida); e (3) quantificação das concentrações dos contaminantes de interesse. A determinação das concentrações dos metais nos alimentos vegetais coletados foi realizada pela técnica de Espectrometria de Absorção Atômica por chama (FAAS) e forno de grafite (GFAAS). Maiores detalhes são apresentados em Magna et al. (2013).

Em relação à exatidão do método, esta foi avaliada de acordo com o material de referência certificado pelo National Institute of Standard and Technology (NIST): folhas de maçã (NIST SRM 1515 Apple leaves) em triplicata para cada batelada de amostras. Ainda, foram realizados testes de recuperação mediante adição de padrão com faixa aceita de $85 \%$ a $115 \%$. Todos os ensaios foram realizados em amostras de polpa do fruto, folhas e/ou caule segundo a espécie analisada, correspondentes 
às suas partes comestíveis. Obtidas as concentrações de ambos os contaminantes, os alimentos vegetais selecionados foram agrupados para sua posterior avaliação em frutas e ervas com o intuito de facilitar a estimativa das doses de exposição devido ao fato das taxas e formas de consumo serem diferenciadas.

\section{População alvo de estudo}

Em relação à população de crianças sob análise, esta foi dividia em duas faixas de idade, de 0 a 6 e de 7 a 17 anos, com o intuito de considerar períodos de exposições diferentes aos contaminantes de interesse. Neste estudo, somente foram consideradas 45 crianças que declararam consumir alimentos vegetais dos seus próprios quintais. Estas tiveram mensurada a sua massa corporal com o uso de balanças do tipo cegonha ou de piso, em suas residências e em presença dos pais ou responsáveis.

Cabe destacar que tanto a aplicação dos questionários quanto a mensuração da massa corporal nas crianças seguiram os princípios éticos estabelecidos pela Resolução 196/96, do Ministério da Saúde, que trata do gerenciamento de pesquisas que envolvem seres humanos, tendo a devida aprovação dos Comitês de Ética em Pesquisa do Centro de Pesquisa Gonçalo Muniz (FIOCRUZ) (Protocolo ${ }^{\circ} 324$, parecer $n^{\circ}$ 217/2010) e da Universidade Católica do Salvador (UCSAL) (Ofício nº 0073/10).

\section{Estimativa das doses de exposição e caracterização do risco}

A estimativa das doses de exposição foi realizada mediante a Equação 1, proposta pela Agency For Toxic Substances and Disease registry (2005):

$\mathrm{D}=\left(\mathrm{C}^{\star} \mathrm{TC} \mathrm{FE}^{\star}\right) / \mathrm{PC}$

onde: D é a dose de exposição $\left(\mathrm{mg} \cdot \mathrm{kg}^{-1} \cdot \mathrm{dia}^{-1}\right)$; C é a concentração do contaminante ( $\left.\mathrm{mg}^{-\mathrm{kg}^{-1}}\right)$; TC é a taxa de consumo (kg.dia $\left.{ }^{-1}\right)$; FE é o fator de exposição (adimensional); e PC é a massa corporal (kg). As doses foram levadas às unidades $\mu \mathrm{g} \cdot \mathrm{kg}^{-1} \cdot \mathrm{dia}^{-1}$ para facilitar a comparação com os valores de referência.

A seguir, apresentam-se as considerações sobre as variáveis utilizadas para a quantificação das doses de exposição aos contaminantes através do consumo de alimentos vegetais.

- Concentração dos contaminantes: os valores de concentração de ambos os contaminantes utilizados na estimativa das doses corresponderam aos valores médios obtidos para cada grupo de alimentos vegetais avaliados (frutas e ervas). Foram empregados os valores médios de ambos os contaminantes, pois dificilmente as crianças estão e estarão expostas aos níveis máximos determinados nos alimentos vegetais.

- Massa corporal: o valor empregado para a variável massa corporal correspondeu à massa corporal média para ambas as faixas de idade avaliadas e considerando apenas as crianças que declararam consumir alimentos vegetais cultivados nos seus quintais.

- Taxa de consumo: para o consumo diário de frutas se considerou o valor de consumo médio da população brasileira determinada na pesquisa de orçamentos familiares (POF 2002-2003) reportada pelo Instituto Brasileiro de Geografia e Estatística (IBGE) de 132 g.dia ${ }^{-1}$ ou 0,132 kg.dia ${ }^{-1}$ e exposta por Pinheiro e Gentil (2005). No caso das ervas foi utilizado como referência o valor em massa de ervas ( $\mathrm{g}$ ) encontrado no conteúdo de chás produzidos industrialmente e se estabeleceu como consumo diário o valor de 5,0 g.dia ${ }^{-1}\left(0,005 \mathrm{~kg} \cdot \mathrm{dia}^{-1}\right)$, considerando uma infusão diária.

- Fator de exposição: o fator de exposição está baseado em um consumo de 7 dias por semana, (48 semanas) em períodos de 6 e 17 anos. O valor do fator de exposição para exposições consideradas como crônicas corresponde à unidade segundo a Agency for Toxic Substances and Disease Registry (2005), valor adotado no presente trabalho.

É importante destacar que a estimação das doses de exposição e, portanto, do risco pelos contaminantes via alimentar, pode ser considerada como conservativa em função dos critérios e valores utilizados para o cálculo das mesmas. Para realizar uma estimativa menos conservativa devem ser considerados, além das variáveis mencionadas anteriormente, outros aspectos tais como período de ocorrência dos alimentos vegetais estudados, o maior consumo de algum alimento vegetal em comparação com outros, entre outras variáveis.

O risco para a saúde das crianças estudadas que impõe a exposição aos metais se caracterizou quantitativamente mediante a estimação do Risco Potencial (RP), dado pela Equação 2, apresentada por Sobrino et al. (2007) e desenvolvida pela USEPA. Considera-se que existe um risco inaceitável para a saúde quando o RP supera a unidade. Deve ser ressaltado aqui, que o presente estudo se ateve na análise da potencial rota de exposição por consumo de alimentos vegetais contaminados, não considerando outras rotas tais como poeira, solo ou outros meios como consumo de água, leite e carne, vias complementares necessárias para obter uma avaliação do risco integral.

$\mathrm{RP}=\mathrm{E} / \mathrm{IDA}$

onde: RP é o Risco Potencial; E é a dose estimada do contaminante ( $\mu \mathrm{g}$. $\left.\mathrm{kg}^{-1} \cdot \mathrm{dia}^{-1}\right)$; e IDA é a Ingestão Diária Admissível $\left(\mu \mathrm{g} \cdot \mathrm{kg}^{-1} \cdot \mathrm{dia}^{-1}\right)$.

Ainda, foi estimada a taxa de consumo (g.dia ${ }^{-1}$ ) para o grupo de alimentos que apresentou o maior risco na avaliação. Esta estimativa foi feita para crianças com massas corporais mínima, média e máxima mensuradas em cada faixa de idade e utilizando as concentrações médias dos contaminantes determinadas nos grupos de alimentos vegetais. A taxa de consumo calculada supõe uma ingestão de alimentos que pode ser considerada segura, não representando um risco sobre a saúde 
das crianças pelos contaminantes de interesse. Para o cálculo da taxa foi utilizada a Equação 3, desenvolvida pela USEPA e apresentada no trabalho de Sobrino et al. (2007) que relaciona a ingestão diária admissível ou a dose de referência dos contaminantes, a massa corporal das crianças e a concentração determinada nos alimentos vegetais.

\section{$\mathrm{TC}=\mathrm{IDA}^{\star} \mathrm{PC} / \mathrm{C}$}

onde: TC é a taxa de consumo de frutas (g.dia $\left.{ }^{-1}\right)$; IDA é a Ingestão Diária Admissível $\left(\mu \mathrm{g} \cdot \mathrm{kg}^{-1} \cdot \mathrm{dia}^{-1}\right)$; PC é a massa corporal; C é a concentração dos contaminantes avaliados.

\section{RESULTADOS E DISCUSSÃO}

\section{Obtenção dos valores das variáveis para o cálculo da exposição}

Os ensaios realizados nas amostras de alimentos vegetais estudadas, num total de 107, apresentaram valores de $\mathrm{Pb}$ variando entre 0,18 e
118,2 mg.kg-1 e valores de Cd entre 0,04 e 7,39 mg.kg-1. Em ambos os casos, os valores obtidos expõem a contaminação das espécies avaliadas acima dos valores limites estabelecidos pela OMS/FAO (0,1 mg.kg-1 para $\mathrm{Pb}$ e $0,05 \mathrm{mg} \cdot \mathrm{kg}^{-1}$ para $\mathrm{Cd}$ ). A Tabela 1 sumariza os resultados.

Os resultados obtidos por grupos (frutas e ervas) apresentaram teores médios de $\mathrm{Pb}$ e Cd em frutas de 20,90 e 0,41 mg.kg-1 , respectivamente. No caso das ervas, o conteúdo médio de $\mathrm{Pb}$ foi de $18,00 \mathrm{mg} \cdot \mathrm{kg}^{-1}$, e de 2,00 mg.kg-1 para o Cd. Na Tabela 2 se apresenta o resumo dos resultados obtidos segundo o grupo de alimentos vegetais analisados.

Resultados sobre a contaminação de alimentos vegetais cultivados na área foram apresentados por Cunha e Araújo (2001), com concentrações de $\mathrm{Pb}$ e $\mathrm{Cd}$ em vegetais comestíveis tais como mamão, goiaba e manga. Foram obtidos valores de $\mathrm{Pb}$ entre 11,9 e 12,6 mg.kg-1. Os valores de concentração de Cd variaram entre 1,84 e 1,91 mg.kg-1 . Concentrações de $\mathrm{Pb}$ mais atualizadas foram apresentadas por Rocha (2010), com teores detectados em alimentos vegetais tais como aipim, manga e couve variando entre 1,2 e 9,5 mg. $\mathrm{kg}^{-1}$. Todos estes valores estão acima das concentrações limites recomendadas pela OMS/FAO para alimentos vegetais. Na Tabela 3 se apresenta um resumo das concentrações médias detectadas por diversos

Tabela 1 - Concentrações de $\mathrm{Pb}$ e Cd detectadas nos alimentos vegetais analisados.

\begin{tabular}{|c|c|c|c|c|c|c|c|c|c|c|c|c|c|}
\hline \multirow{2}{*}{\multicolumn{2}{|c|}{ Espécies vegetais }} & \multicolumn{6}{|c|}{$\mathrm{Pb}\left(\mathrm{mg} \cdot \mathrm{kg}^{-1}\right)$} & \multicolumn{6}{|c|}{$\mathrm{Cd}\left(\mathrm{mg} \cdot \mathrm{kg}^{-1}\right)$} \\
\hline & & \multirow{2}{*}{$\begin{array}{l}\text { Min } \\
0,18\end{array}$} & \multirow{2}{*}{$\begin{array}{l}\text { Max } \\
0,24\end{array}$} & \multirow{2}{*}{$\begin{array}{l}\text { Média } \\
\text { 0,20 }\end{array}$} & \multirow{2}{*}{$\begin{array}{l}\text { DP } \\
\text { 0,11 }\end{array}$} & \multirow{2}{*}{$\begin{array}{l}n \\
9\end{array}$} & \multirow{2}{*}{$\begin{array}{c}n_{m} \\
4\end{array}$} & \multirow{2}{*}{$\begin{array}{l}\text { Min } \\
0,04\end{array}$} & \multirow{2}{*}{$\begin{array}{l}\text { Max } \\
2,2\end{array}$} & \multirow{2}{*}{$\begin{array}{c}\text { Média } \\
\text { 0,82 }\end{array}$} & \multirow{2}{*}{$\begin{array}{l}\text { DP } \\
1,22\end{array}$} & \multirow{2}{*}{$\begin{array}{l}n \\
9\end{array}$} & \multirow{2}{*}{$\begin{array}{l}n_{m} \\
4\end{array}$} \\
\hline 1 & Banana (Musa paradisica) & & & & & & & & & & & & \\
\hline 2 & Acerola (Malpichia glabral) & 1,10 & 118,2 & 36,7 & 42,6 & 10 & 6 & 0,22 & 0,57 & 0,33 & 0,16 & 10 & 4 \\
\hline 3 & Manga (Mangifera indica) & 0,36 & 22,2 & 10,8 & 9,8 & 12 & 7 & 0,09 & 0,37 & 0,22 & 0,09 & 12 & 6 \\
\hline 4 & Goiaba (Psidum cattleianum) & $<4$ & 27,4 & * & * & 5 & * & 0,11 & $<2$ & * & * & 5 & * \\
\hline 5 & Limão (Citrus limonum) & 6,95 & 32,5 & 21,2 & 2,96 & 6 & 4 & 0,04 & 0,1 & 0,06 & 0,03 & 6 & 4 \\
\hline 6 & Laranja (Citrus aurantium) & 0,47 & 117,3 & 39,9 & 40,4 & 11 & 6 & 0,08 & 0,15 & 0,12 & 0,04 & 9 & 4 \\
\hline 7 & Mandioca (Manihot esculenta) & 10,7 & 23 & 16,8 & 8,7 & 4 & 4 & 0,61 & 1,25 & 0,93 & 0,45 & 2 & 2 \\
\hline 8 & Aroeira (Schinus molle) & 1,49 & 44,8 & 14,8 & 14,0 & 11 & 8 & 0,46 & 0,79 & 0,62 & 0,18 & 11 & 4 \\
\hline 9 & Cana (Arundo donax. L) & 1,61 & $<4$ & * & * & 8 & * & 1,66 & $<2$ & * & * & 8 & * \\
\hline 10 & Cidreira (Lippia alba) & 1,26 & 32,3 & 16,8 & 12,3 & 10 & 8 & 0,25 & 4,95 & 1,35 & 2,01 & 10 & 5 \\
\hline 11 & Capim santo (Cybopogon citratus) & 13,1 & 23 & 16,7 & 6,3 & 8 & 4 & 0,1 & 6,68 & 2,84 & 3,42 & 8 & 4 \\
\hline 12 & Alumã (Veronia bahiensis tol) & 6,58 & 51,7 & 24,9 & 7,1 & 9 & 4 & 0,75 & 7,39 & 3,21 & 3,63 & 9 & 4 \\
\hline 13 & Boldo do Chile (Peumus boldus molina) & 14,6 & 19 & 16,8 & 3,1 & 4 & 2 & $<5$ & $<5$ & * & * & 4 & * \\
\hline
\end{tabular}

*Valores indeterminados por estarem abaixo do limite de detecção do método empregado (FAAS). n: número de ensaios; DP: desvio padrão em função do número de ensaios; n : número de amostras com resultados acima do limite de detecção utilizadas para o cálculo dos valores médios. No caso do boldo do Chile não foi quantificado o metal Cd mediante GFAAS. No caso do fruto goiaba somente um valor apresentou conteúdo mensurável (GFAAS)

Tabela 2 - Concentração média de Pb e Cd segundo grupo de alimentos vegetais.

\begin{tabular}{|c|c|c|c|c|c|}
\hline Grupos & $\mathrm{Pb}\left(\mathrm{mg} \cdot \mathrm{kg}^{-1}\right)$ & $\mathrm{DP}\left(\mathrm{mg} \cdot \mathrm{kg}^{-1}\right)$ & $\mathrm{Cd}\left(\mathrm{mg} \cdot \mathrm{kg}^{-1}\right)$ & $\mathrm{DP}\left(\mathrm{mg} \cdot \mathrm{kg}^{-1}\right)$ & $\mathrm{n}$ \\
\hline Frutas & 20,9 & 15,2 & 0,41 & 0,33 & 57 \\
\hline Ervas & 18,0 & 3,9 & 2,0 & 1,06 & 50 \\
\hline
\end{tabular}

DP: desvio padrão; n: número de ensaios. 
estudos em Santo Amaro, Bahia, além dos detectados no presente trabalho e os valores limites estabelecidos pela OMS/FAO para ambos os contaminantes. Pode-se perceber a persistência da contaminação por $\mathrm{Pb}$ e Cd nos alimentos vegetais cultivados na área de estudo. Contudo, uma comparação entre elas fica dificultada visto que as medidas foram feitas em espécies diferentes e coletadas em pontos distintos da área atingida. Cabe ressaltar que as mais altas concentrações medidas neste estudo são em decorrência das espécies analisadas serem cultivadas em solos comprovadamente contaminados. Também é importante destacar que o valor máximo obtido neste estudo para $\mathrm{Pb}$ foi de $118,8 \mathrm{mg} \cdot \mathrm{kg}^{-1} \mathrm{e}$ para cádmio de 7,39 mg.kg-1, concentrações menores que as obtidas por Tavares e Carvalho (1992) em alimentos vegetais durante o último ano do funcionamento da fábrica (215 mg.kg-1 para $\mathrm{Pb}$ e 11,8 mg.kg ${ }^{-1}$ para Cd).

Em relação ao consumo de vegetais, das 101 crianças que participaram na aplicação do questionário, 44,5\% $(\mathrm{n}=45)$ declararam consumir frutas e ervas cultivadas nos quintais de suas casas. Estas 45 crianças e adolescentes informaram que a forma de consumo é diversa, variando desde fruto completo (forma preferencial) a sucos e frutos misturados na comida. No caso das ervas, 32,6\% das crianças avaliadas declararam consumi-las, sendo a infusão a principal forma de ingestão. Com respeito à frequência de consumo, os resultados obtidos apresentaram um consumo de sete vezes na semana, valor representado pela moda, seguido pelo consumo de três vezes na semana. Estes resultados confirmam o consumo tanto de frutas quanto de ervas pelas crianças residentes na área contaminada.

O valor da massa corporal média obtido para crianças na faixa de 0 a 6 anos foi de $16,4 \mathrm{~kg}$, com desvio padrão de 4,1 kg $(\mathrm{n}=13)$. Já a massa corporal média das 32 crianças pertencentes à segunda faixa etária (7 a 17 anos) foi de 42,4 kg, com um desvio padrão de 14,4 kg. Vale ressaltar que os resultados obtidos para esta variável caracterizam as crianças da área de estudo, considerando fatores próprios dessa população tais como raça, estado nutricional e condições socioeconômicas. A Tabela 4 apresenta um resumo dos valores utilizados para a análise da exposição da população aos contaminantes de interesse.

Crianças de 0 a 6 anos de idade apresentaram um valor médio de dose de exposição de $166,9 \mu \mathrm{g} \cdot \mathrm{kg}^{-1} \cdot \mathrm{dia}^{-1}$ de $\mathrm{Pb}$ por consumo de frutas. Este resultado supera em aproximadamente 48 vezes o valor da IDA recomendado pela OMS/FAO (3,5 $\left.\mu \mathrm{g} \cdot \mathrm{kg}^{-1} \cdot \mathrm{dia}^{-1}\right)$, assim como os valores apresentados por Castell et al. (2005) e Santos et al. (2004), expostos na Tabela 5. Para a segunda faixa de idade, o valor médio estimado foi de $64,9 \mu \mathrm{g} \cdot \mathrm{kg}^{-1}$. dia ${ }^{-1}$, aproximadamente 20 vezes o valor de referência da OMS/FAO. Neste ponto vale destacar que, considerando a dispersão encontrada nos resultados experimentais de concentração de metais nas frutas (ver Tabela 2), as diferenças nos valores de massa corporal e o fato do consumo diário de frutas ter sido adotado de dados nacionais, fatores que dão às doses de exposição obtidas um caráter majoritariamente estimativo, estas incertezas não são suficientes para justificar uma diferença de 48 ou 20 vezes na taxa de ingestão com relação à máxima IDA recomendada pela OMS/FAO.

Tabela 3 - Concentrações médias de $\mathrm{Pb}$ e $\mathrm{Cd}$ em alimentos vegetais obtidas em diversos estudos em Santo Amaro, Bahia, e os limites máximos permitidos pela OMS/FAO.

\begin{tabular}{l|c|c} 
Autores & $\mathrm{Pb}\left(\mathrm{mg}^{\mathrm{kg}}{ }^{-1}\right)$ & $\mathrm{Cd}\left(\mathrm{mg}_{\mathrm{kg}}{ }^{-1}\right)$ \\
\hline Cunha e Araújo (2001) & 12,2 & 1,87 \\
\hline Rocha (2010) & 3,6 & $*$ \\
\hline Presente estudo & 19,6 & 1,05 \\
\hline OMS/FAO (2009) & 0,1 & 0,05 \\
\hline
\end{tabular}

*Elemento não analisado no estudo

Tabela 4 - Variáveis de estimação das doses de exposição aos contaminantes $\mathrm{Pb}$ e $\mathrm{Cd}$ por consumo de alimentos vegetais.

\begin{tabular}{l|c|c}
\multirow{2}{*}{ Variáveis } & \multicolumn{2}{|c}{ Faixa etária } \\
\cline { 2 - 3 } & 0 a 6 & 7 a 17 \\
Massa corporal média (kg) & 16,4 & 42,4 \\
\hline Consumo diário de ervas (kg.dia') & \multicolumn{2}{|c}{0,005} \\
\hline Consumo diário de frutas (kg.dia') & \multicolumn{2}{|c}{0,132} \\
\hline Frequência de exposição (dias.anos') & \multicolumn{2}{|c}{365} \\
\hline Duração da exposição (anos) & 6 & 17 \\
\hline
\end{tabular}

Tabela 5 - Doses de exposição ao Pb e Cd por consumo alimentar de frutas e ervas.

\begin{tabular}{|c|c|c|c|c|c|c|}
\hline Fonte & Faixa de idade & \multicolumn{2}{|c|}{ Dose Pb ( $\left.\mu \mathrm{g} . \mathrm{kg}^{-1} \cdot \mathrm{dia}^{-1}\right)$} & \multicolumn{2}{|c|}{ Dose Cd ( $\left.\mu \mathrm{g} \cdot \mathrm{kg}^{-1} \cdot \mathrm{dia}^{-1}\right)$} & Local \\
\hline \multirow{2}{*}{ Presente estudo } & Oa 6 & 166,9 & 5,44 & 2,95 & 0,58 & \multirow{2}{*}{ Santo Amaro, Bahia, Brasil } \\
\hline & $7 a 17$ & 64,96 & 2,12 & 1,15 & 0,22 & \\
\hline Rocha (2010) & Adultos & \multicolumn{2}{|c|}{36,0} & \multicolumn{2}{|c|}{ * } & Santo Amaro, Bahia, Brasil \\
\hline Santos et al. (2004) & População geral & \multicolumn{2}{|c|}{0,40} & \multicolumn{2}{|c|}{0,02} & Rio de Janeiro, Brasil \\
\hline
\end{tabular}

*Dose de exposição não estimada para este contaminante. 
Para o caso do Cd, os valores médios estimados para as doses de exposição foram de $2,95 \mu \mathrm{g} \cdot \mathrm{kg}^{-1} \cdot \mathrm{dia}^{-1}$ (0 a 6 anos) e $1,15 \mu \mathrm{g} \cdot \mathrm{kg}^{-1} \cdot \mathrm{dia}^{-1}$ (7 a 17 anos), superiores ao valor da IDA (OMS/FAO) de 1,0 $\mu \mathrm{g} \cdot \mathrm{kg}^{-1}$. $\mathrm{dia}^{-1} \mathrm{e}$ aos valores apresentados por outros autores (Tabela 5).

Em relação às doses de exposição estimadas para $\mathrm{Pb}$ devido ao consumo de ervas, somente a primeira faixa de idade supera a IDA recomendada pela OMS/FAO em aproximadamente 35,7\%. Este fato pode ter explicação principalmente pela influência da massa corporal e a menor taxa de consumo de ervas em comparação com as frutas. As doses de Cd estimadas por consumo alimentar de ervas encontram-se abaixo do valor da IDA e na faixa de valores estimados por Castell et al. (2005) na Espanha, em população de crianças e adolescentes residentes em áreas sem contaminação. Deve-se destacar, contudo, que as doses de exposição a serem consideradas para a população correspondem sempre à soma das doses obtidas para frutas e ervas (e para outros alimentos não considerados neste estudo), de forma que estas são sempre superiores aos valores de IDA considerados como aceitáveis.

Os valores das doses de exposição ao $\mathrm{Pb}$ e $\mathrm{Cd}$ por consumo de alimentos vegetais cultivados em quintais da Rua Rui Barbosa merecem especial preocupação, visto que as crianças correspondem à população mais susceptível à contaminação, pois absorvem mais o $\mathrm{Pb}$ que ingerem comparativamente aos adultos. Estima-se que aproximadamente $73 \%$ do $\mathrm{Pb}$ que entra no corpo das crianças é acumulado nos ossos (AGENCY FOR TOXIC SUBSTANCES AND DISEASE REGISTRY, 2007). Além disso, crianças podem absorver e reter maior quantidade de $\mathrm{Pb}$ devido às diferenças fisiológicas e metabólicas, absorvendo aproximadamente $50 \%$ do contaminante na dieta alimentar, enquanto os adultos absorvem aproximadamente 10\% (KABATA-PENDIAS \& MUKHERJEE, 2007).

Em relação ao Cd, a principal rota de absorção deste contaminante em populações expostas ambientalmente é pela via digestiva, de grande importância devido à possibilidade de bioacumulação do metal nos diversos níveis tróficos. Através dessa rota, a absorção do metal se dá de forma lenta, variando em torno de 3 a 10\% do total ingerido. No entanto, existem fatores que podem alterar essa absorção, como o tipo de dieta adotada e as condições nutricionais da população exposta, pois alguns minerais podem participar como elementos que dificultam a absorção do cádmio (SANTA MARINHA, 2011). Neste contexto, reveste-se de uma importância adicional a estimativa das doses de exposição em um cenário considerado como crítico pela persistência da contaminação no solo e nos alimentos vegetais cultivados, pelo consumo dos mesmos e pelas características particulares das crianças avaliadas.

O RP pela exposição ao $\mathrm{Pb}$ e Cd pelas crianças foi determinado para ambos os grupos de alimentos vegetais e ambas as faixas de idade. Os valores obtidos são apresentados na Figura 2. Os maiores valores de $\mathrm{RP}$ ao $\mathrm{Pb}$ foram obtidos para a faixa de idade de 0 a 6 anos por consumo de frutas e ervas, com índices de 47,69 e 5,45, respectivamente. Valores de RP maiores que a unidade implicam em um risco inadmissível ao contaminante pela população. Somente no cenário onde se considera o consumo de ervas isolado, para a faixa etária de 7 a 17 anos, é que o RP da exposição ao $\mathrm{Pb}$ pode ser considerado como aceitável. No caso da exposição ao Cd por consumo de ervas o risco pode ser considerado como aceitável em ambas as faixas de idade avaliadas.

O RP para ambos os contaminantes em relação ao consumo de frutas é inaceitável, portanto, é recomendado não consumir frutas cultivadas nos quintais das casas da área de estudo.

A Tabela 6 apresenta as taxas máximas admissíveis de consumo de frutas para a população estudada e em função das massas corporais (mínimo, médio e máximo obtido para a população de estudo) e o consumo de frutas. Conforme se pode observar, os percentuais de $\mathrm{Pb}$ nos alimentos limitam a taxa de consumo de frutas a valores muito inferiores ao padrão determinado para a população brasileira, de $132 \mathrm{~g} \cdot \mathrm{dia}^{-1}$. A restrição ao consumo de frutas cultivadas nos quintais da área de estudo pela população se faz mais evidente quando se considera o consumo de alimentos vegetais recomendado pela OMS de 400 g.dia ${ }^{-1}$ (PINHEIRO \& GENTIL, 2005).
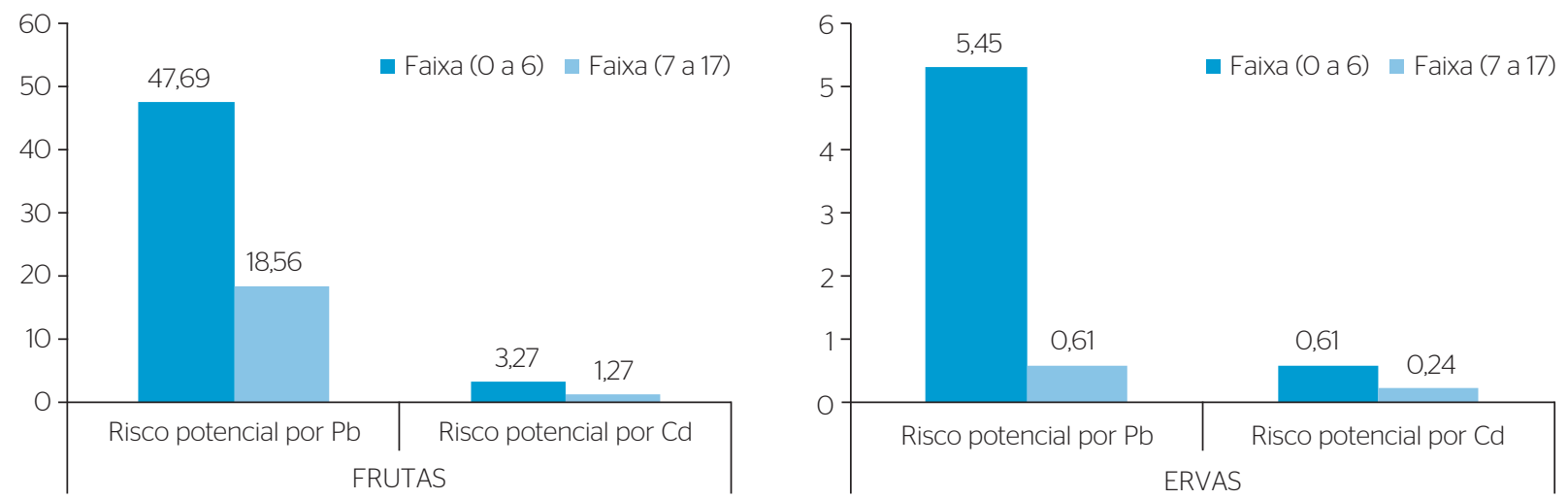

Figura 2 - Risco potencial ao $\mathrm{Pb}$ e Cd por consumo de frutas (A) e risco potencial ao $\mathrm{Pb}$ e $\mathrm{Cd}$ por consumo de ervas (B). 
Tabela 6 - Taxa de ingestão de frutas admissível para a população estudada (g.dia-1).

\begin{tabular}{l|c|c|c}
\multirow{2}{*}{$\begin{array}{l}\text { Faixa } \\
\text { etária } \\
\text { (anos) }\end{array}$} & $\begin{array}{c}\text { Massa } \\
\text { corporal } \\
(\mathrm{kg})\end{array}$ & \multicolumn{2}{|c}{$\begin{array}{c}\text { Taxa de consumo segura para Frutas } \\
\text { (g.dia') }\end{array}$} \\
\cline { 3 - 4 } & 7,0 & $\mathrm{~Pb}$ & $\mathrm{Cd}$ \\
\cline { 2 - 4 } O a 6 & 16,4 & 1,17 & 18,9 \\
\cline { 2 - 4 } & 22,9 & 3,74 & 44,32 \\
\hline \multirow{4}{*}{ 7a 17 } & 13,20 & 2,83 & 61,89 \\
\cline { 2 - 4 } & 42,4 & 7,10 & 35,67 \\
\cline { 2 - 4 } & 77,0 & 12,89 & 114,59 \\
\hline
\end{tabular}

Peso corporal mínimo; médio e máximo determinado em cada faixa etária; valor de concentraçăo médio de Pb e Cd utilizados para o cálculo das taxas de consumo de segurança para a população de crianças de Santo Amaro.

\section{CONCLUSÕES}

As doses de exposição ao $\mathrm{Pb}$ e Cd estimadas, considerando-se a rota de contaminação por consumo de alimentos vegetais, podem ser consideradas como elevadas e preocupantes em função do fato de que os valores obtidos superam, com folga, os valores de IDA estabelecidos pela OMS/FAO para ambos os contaminantes. Deve-se ressaltar aqui que o presente estudo considera apenas as doses dos contaminantes por consumo de alimentos vegetais, excluindo-se os valores de ingestão calculados para outros grupos alimentícios tais como carne, leite e água.

Atenção especial deve ser devotada às crianças com algum grau de desnutrição, em ambas as faixas etárias, pois que esta condição aumenta a absorção dos contaminantes.
Variáveis tais como a taxa e a frequência de consumo dos alimentos vegetais correspondem a fatores de especial preocupação, influenciando de forma significativa os valores estimados para as doses de exposição. Estes fatores podem ser controlados através de medidas restritivas, tais como evitar o consumo dos alimentos vegetais cultivados nos quintais de casas; avaliação da condição nutricional da dieta da população e sua posterior melhora visando à diminuição da absorção dos metais pelas crianças.

Tanto os valores das doses de exposição calculadas, do $\mathrm{RP}$ para $\mathrm{Pb}$ e $\mathrm{Cd}$ em frutas e ervas e das taxas de consumo consideradas como seguras para as crianças permitem concluir que o consumo de alimentos vegetais cultivados em quintais no entorno da COBRAC corresponde a uma rota de exposição ativa aos contaminantes analisados e que deve ser debelada.

Em suma, pode-se dizer que os resultados obtidos permitem concluir que um grande número de crianças estão expostas aos contaminantes $\mathrm{Pb}$ e $\mathrm{Cd}$ pelo consumo de alimentos vegetais cultivados nos quintais de suas residências. Essa via de exposição representa um risco real que deve ser considerado na tomada de decisões para mitigação do problema em curto e longo prazo, devido ao caráter acumulativo e aos potenciais efeitos adversos destes elementos nos seres humanos em longo prazo, quando considera-se a sua persistência e os altos níveis detectados nos alimentos vegetais, além do consumo dos mesmos.

\section{AGRADECIMENTOS}

A realização deste trabalho foi possível graças ao apoio das equipes do Centro de Pesquisas e Desenvolvimento (CEPED) ao Laboratório de geotecnia ambiental da UFBA (GEOAMB), a população de Santo Amaro-BA e as instituições CAPES, Fundação Escola Politécnica e a Secretaria de Ciência, Tecnologia e Inovação do Estado da Bahia (SECTI-BA)

\section{REFERÊNCIAS}

AGENCY FOR TOXIC SUBSTANCES AND DISEASE REGISTRY. (2005) Public Health Assessment: guidance manual. Atlanta, EUA. 357p.

AGENCY FOR TOXIC SUBSTANCES AND DISEASE REGISTRY. (2007) ToxGuide for Lead. Disponível em: <http://www.atsdr.cdc.gov/es/phs/ es_phs13.html>. Acesso em: 14 set. 2009.

AGENCY FOR TOXIC SUBSTANCES AND DISEASE REGISTRY. (2O12) ToxGuide for Cadmium. Disponível em: <http://www.atsdr.cdc.gov/es/ phs/es_phs5.pdf>. Acesso em 14 set. 2009.

ANJOS, J.Â.S.A. (2003) Avaliação da eficiência de uma zona alagadiça (wetland) no controle da poluição por metais pesados: o caso da plumbum em Santo Amaro da Purificação/BA. 301f. Tese (Doutorado em Engenharia) - Universidade de São Paulo, São Paulo.
BRASIL (2009) Resolução N420, de 28 de dezembro de 2009 Conselho Nacional do Meio Ambiente. Diário Oficial União, Brasília, DF, 30 dez, no 249, p. 81-84. Disponível em: <http:// www.mma.gov.br/port/conama/legiabre.cfm?codlegi=620> Acesso em: 16 jun. 2010.

CASTELL, G.V.; RAFAEL, P.G.; ALONSO, T.I.; CATALANN, G.J.; MALLAFRÉ, L.M.J.; ROIG, D.L.J.; CID, R.S. (2005) Contaminantes Químicos. Estúdio de Dieta Total en Cataluña. Barcelona: Agencia Catalana de Seguridad Alimentaria. $127 \mathrm{p}$

CHARY, N.S.; KAMALA, C.T.; RAJ SUMAN SAMUEL, D. (2008) Assessing risk of heavy metals from consuming food grow on sewage irrigated soils and food chain transfer. Ecotoxicology and Environmental Safety, v. 69, n. 3, p. 513-24. 
CUNHA, F.G. (2003). Contaminação Humana e Ambiental por Chumbo no Vale do Ribeira, nos Estados de São Paulo e Paraná, Brasil. 111 f. Tese (Doutorado em Ciências). Instituto de Geociências, Universidade Estadual de Campinas, Campinas.

CUNHA, F.G.; FIGUEIREDO, B.R.; PAOLIELLO, M.M.B.; de CAPITANI, E.M.; SAKUMA, A.M. (2005) Human and environmental lead contamination in the Uppar Ribeira Valley, Southeastern Brazil. Terrae, v. 2, n. 1-2, p. 28-36.

CUNHA, P.S.P \& ARAÚJO, P.S.P. (2001) Laudo Pericial de Avaliação e Quantificação da Contaminação Ambiental por Chumbo e Cádmio no Município de Santo Amaro da Purificação-Estado da Bahia. 167 p.

FREITAS, C.U.; CAPITANI, E.M.; GOUVEIA, N.; SIMONETTI, M.H.; SILVA, M.R.P.; KIRA, C.S.; SAKUMA, A.M.; CARVALHO, M,F.H.; DURAN, M.C.; TIGLEA, P.; ABREU, M.H. (2007) Lead exposure in an urban community: investigation of risk factors anda assessment of the impact of lead abatement measures. Environment Research, v. 103, n. 3, p. 338-344.

FUNASA - FUNDAÇÃO NACIONAL DE SAÚDE. (2003) Avaliação de risco à saúde por exposição a metais pesados em Santo Amaro da Purificação, Bahia. Disponível em: <http://www.acpo.org.br/ saudeambiental/CGVAM/O2_Avaliaca_de_Risco/05_santo_amaro_ ba/>. Acesso em: 8 maio 2009.

KABATA-PENDIAS, A. \& MUKHERJEE, B.A. (2007) Trace Elements From Soil to Human. New York: Springer. 561 p.

KABATA-PENDIAS, A. \& PENDIAS, H. (2001) Trace Elements in Soils and Plants. 3 ed. Florida: CRC press. $432 \mathrm{p}$

MACHADO, S.L.; RIBEIRO, L.D.; KIPERSTOK, A.; BOTELHO, M.A.B.; CARVALHO, M. de F. (2004) Diagnóstico da contaminação por metais pesados em Santo Amaro-BA. Revista de Engenharia Sanitária e Ambiental, v. 9, n. 2, p. 140-55

MACHADO, S.L (Coord.). (2003) Projeto PURIFICA: Proposta para remediação de áreas impactadas pela atividade extrativa de chumbo em Santo Amaro, Bahia: Relatório Síntese. Disponível em: <http:// www.geoamb.eng.ufba.br/site/sites/default/files/arquivos/relatorios/ principaisconclusoespurifica.pdf >. Acesso em: 10 maio 2009.

MACHADO, S.L.; RABELLO, T.S.; PORTELLA, R.B.; CARVALHO, M.F;; MAGNA, G.M.M. (2013). A study of the routes of contamination by lead and cadmium in Santo Amaro, Brazil. Environmental Technology, v. 34, n. 5, p. 559-571.

MAGNA, G.A.M; MACHADO, S.L; PORTELLA, R.B; CARVALHO, M.F. (2O13) Chumbo e cádmio detectados em alimentos vegetais e gramíneas no município de Santo Amaro-Bahia. Química Nova, v. 36, n. 7. p. 989-997.

MOREIRA, F.R. \& MOREIRA, J.C. (2004) Os efeitos do chumbo sobre o organismo humano e seu significado para a Saúde. Revista Panamericana de Salud Publica, v. 15, n. 2, p. 119-29.

OMS - ORGANIZAÇÃO MUNDIAL DA SAÚDE. (2009) Comité Codex Alimentarius. Norma general del Codex para los contaminantes y las toxinas presentes en los alimentos y piensos. Codex Stan 193-1995, Rev. 4-2009. Disponível em: <http:// www.codexalimentarius.net/download/ standards/17/CXS_193s.pdf>. Acesso em: 30 maio 2009.
PAOLIELLO, M.M.; CAPITANI, E.M.; CUNHA, F.G.; MATSUO, T.; CARVALHO, M.F.; SAKUMA, A.; FIGUEIREDO, B.R. (2002) Exposure of children to lead and cadmium from a mining area of Brazil. Environmental Research, v. 88, n. 2, p. $120-128$

PINHEIRO, A.R.O. \& GENTIL, P.C.H. (2005) A iniciativa de incentivo ao consumo de frutas, verduras e legumes: uma estratégia para abordagem intersetorial no contexto da segurança alimentar e nutricional. CONSEA-Brasil. Disponível em: <http://dtr2004. saude.gov.br/nutricao/documentos/iicflv_br_consea.pdf>. Acesso em: 12 out. 2009.

PORTELLA, R.B. (2007) Desarrollo y evaluación de métodos integrados para la gestión de suelos y aguas subterráneas contaminadas aplicando metodología de análisis de riesgo sobre salud humana. $271 \mathrm{f}$. Tese (Doctorado en Ingeniería Ambiental) - Universidad Politécnica de Cataluña, Cataluña

RABELO, T.S. (2010) Estudo das rotas remanescentes de contaminação por chumbo e cádmio no município de Santo Amaro-BA. 154 f. Dissertação (Mestrado em Engenharia Ambiental Urbana) - Universidade Federal da Bahia, Salvador.

RIBEIRO, L.D.; MACHADO, S.L.; KIPERSTOK, A.; BAKER, F.R. (2003) Contaminação por metais pesados em Santo Amaro da PurificaçãoBA: uso de técnicas de hidrometalurgia no reprocessamento de resíduos perigosos. In: $V$ Congresso Brasileiro de Geotecnia Ambiental, Anais... Porto Alegre: PUCRS, p. 140-55.

ROCHA, A.L.S. (2010) Contaminação de alimentos por chumbo em Santo Amaro Bahia, Uma análise retrospectiva. 66 f. Monografia (Curso de Farmácia) - Faculdade de Tecnologia e Ciência, Salvador, Bahia.

SANTA MARINHA, M.S. (2011) Níveis de Cádmio em Sangue e Urina dos Habitantes do Entorno de uma Reserva Ecológica no Pantanal Mato-Grossense. 70 f. Dissertação (Mestrado em Ciências na área de Saúde Publica) - Escola Nacional de Saúde Publica Sergio Arouca, Fundação Oswaldo Cruz, Rio de Janeiro, Rio de Janeiro.

SANTOS, E.E.; LAURIA, D.C.; PORTO DA SILVEIRA, C.L. (2004) Assessment of daily intake of trace elements due to consumption of foodstuffs by adult inhabitants of Rio de Janeiro City. Science of the Total Environmental, v. 327, n. 1-3, p. 69-79.

SOBRINO, F.A.; CACERES, M.C.; ROSAS, C.R. (2007) Evaluación del riesgo por consumir moluscos contaminados con cádmio, cromo y plomo. Hidrobiologica, v. 17, n. 1, p. 49-58.

TAVARES, T.M. \& CARVALHO, F.M. (1992). Avaliação da exposição de populações humanas a metais pesados no ambiente: Exemplos no Recôncavo Baiano. Química Nova, v. 5, n. 2, p. 147-153.

TOBAR, R.M. (2006) Desarrollo y validación de una herramienta de cálculo de riesgo en suelos y aguas subterráneas contaminados con compuestos inorgánicos. 94 f. Dissertação (Maestría en Ingeniería Ambiental) - Universidad Politécnica de Cataluña, Cataluña. 